\title{
HÁBITAT REPRODUCTIVO Y ESTIMACIÓN DEL TAMAÑO POBLACIONAL DEL PINGÜINO DE MAGALLANES (SPHENISCUS MAGELLANICUS) EN ISLA RUPERT, PARQUE MARINO FRANCISCO COLOANE, ESTRECHO DE MAGALLANES.
}

\author{
BREEDING HABITAT AND ESTIMATION OF POPULATION SIZE OF THE \\ MAGELLANIC PENGUIN (SPHENISCUS MAGELLANICUS) ON RUPERT ISLAND, \\ FRANCISCO COLOANE MARINE PARK, STRAITS OF MAGELLAN.
}

Marcela Mirandaa), Jorge Gibbons ${ }^{\mathrm{a}, \mathrm{b})}$, Jaime Cárcamo ${ }^{\text {a) }}$ \& Yerko A. Vilinac(,d)

La selección de los sitios de nidificación en especies de aves nidícolas en ambientes de climas extremos es particularmente relevante, más aún cuando se trata de especies con cuidado parental prolongado, como es el caso de los pingüinos (Sphenisciformes). En el caso del pingüino de Magallanes, Spheniscus magellanicus, Capurro et al. (1988) sugieren que la topografía y el tipo de sustrato serían determinantes en el lugar donde se construyen los nidos. Anteriormente, Boswall \& Maclver (1975) habían sugerido que la pendiente del área era también relevante, evitando construir nidos en lugares planos. García et al. (2002) encontraron que la presencia y densidad de nidos se correlacionaba significativamente con una mayor cobertura de la vegetación.

El pingüino de Magallanes se distribuye a través del litoral del cono sur de Sudamérica desde aproximadamente $30^{\circ}$ de latitud sur en el océano Pacífico
(Chile), alrededor del cabo de Hornos y hasta $42^{\circ} \mathrm{S}$ en el Atlántico (Argentina) incluyendo las islas Malvinas o Falklands. Según los censos realizados por Schiavini et al. (2005) en las 63 colonias reproductivas conocidas de la costa atlántica de Argentina, el tamaño de sus poblaciones se estima en unos 950.000 individuos. Para las islas Malvinas, según los censos realizados por Bingham et al. (2002) en los años 99/2000, su tamaño sería de 150.000 individuos.

A pesar que Magallanes sería el centro principal de la especie en Chile (Venegas 1978), las únicas estimaciones actualizadas que se conocen, son los correspondientes a isla Magdalena con 63.000 parejas reproductivas en el año 2007 y 25.000 parejas en isla Contramaestre en el año 2002 (Bingham \& Martin 2008) y el de la pingüinera de seno Otway con 10.833 individuos en la temporada 2003/2004 (Téllez 2005)․․ Otras colonias reproductivas conoci-

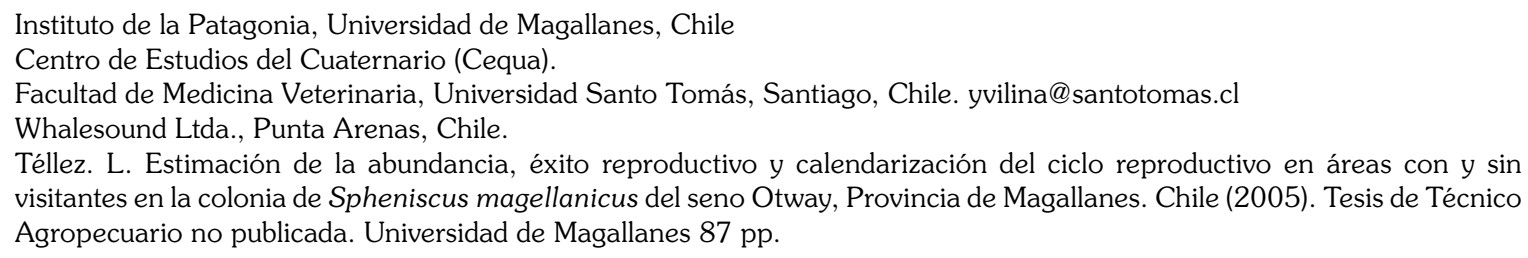


das en el área, pero en las que no se han realizado censos, son isla Marta, en el centro del estrecho de Magallanes, canal Gabriel, isla Carolina, isla Ildefonso $e$ isla Hornos, ubicadas al oeste de los fiordos patagónicos y fueguinos (Venegas 1978, Pisano 1981). En isla Noir Venegas (1999), y luego Kusch et al. (2007) estiman su población reproductiva por sobre las 35.000 parejas y en islas Diego Ramírez Schlatter \& Riveros (1997) estiman 400 individuos.

Este trabajo tiene como objetivo estimar el tamaño de la población y describir el hábitat utilizado por la colonia reproductiva del pingüino de Magallanes en isla Rupert, comparando esta información con un estudio no publicado, realizado en el año 1989 por Soto $(1990)^{2}$. Con ello se busca hacer un aporte al escaso conocimiento de la especie, dado que la mayoría de los estudios sobre las características del hábitat reproductivo se han realizado en ambientes más secos y templados de Argentina, donde domina la vegetación arbustiva (Boswall \& Maclver 1975, Capurro et al. 1988, Gandini et al. 1997, García et al. 2002) y escasamente en ambientes lluviosos, característicos de los fiordos patagónicos. Para ambientes lluviosos, pero de la costa expuesta del sur de Chile existe una breve descripción del hábitat reproductivo de la colonia de isla Puñihuil (Simeone \& Schlatter 1998).

\section{Área de estudio}

Isla Rupert (5346’ S - 72¹3’ W) está emplazada en el estrecho de Magallanes a más de $148 \mathrm{~km}$ al suroeste de la ciudad de Punta Arenas y a unos 2,5 $\mathrm{km}$ al suroeste de la isla Carlos III, dentro del Parque Marino Francisco Coloane. Actualmente se encuentra bajo la administración del Ministerio de Bienes Nacionales de Chile. Presenta una superficie aproximada de 72 hectáreas y se caracteriza por poseer una topografía ondulada, con pendientes fuertes de baja altura. El suelo es de contextura turbosa con pequeños afloramientos rocosos en las partes altas. La vegetación de la isla está caracterizada por la presencia de bosques perennifolios, dominados por el coigüe (Nothofagus betuloides) y el canelo (Drymis winterii) que se extienden en su

2 Soto, N. Proyecto de protección y manejo de las colonias de pingüinos presentes en isla Rupert e isla Recalada, Reserva Nacional Alacalufes.1990. Informe de temporada 1989 - 1990 CONAF, 29 pp perímetro, excepto en la cara norte de la isla. También se encuentra leñadura (Maytenus magellanica). Entre las especies de matorral que se destacan, se encuentran el michay (Berberis ilicifolia), chaura (Gaultheria mucronata), romerillo (Chiliotrichium diffusum), murtilla (Empetrum rubrum), senecios (Lebetanthus myrsinites y Desfontainia spinosa.) y el coicopihue (Philesia magellanica). En el estrato herbáceo destacan los helechos (Blechnum magellanicum), hepáticas, musgos y líquenes. También están presentes en toda la isla los turbales graminoides, destacándose las especies Shoenus antarcticus, Cortaderia pilosa, Carpha alpina y cortadera (Poa flabellata).

Cobertura de la vegetación en isla Rupert.

Para la caracterización de las unidades de vegetación de isla Rupert se analizaron e interpretaron fotos en papel blanco y negro de la isla de 2004 y 2005, con un estereoscopio, además de contar con la ayuda de fotografías digitales a color del perímetro y panorámicas de la isla de 2006. Los límites y áreas de coberturas de las comunidades identificadas se establecieron utilizando el programa Arc View 3.2.

\section{Colonias de pingüinos}

Entre los días 26 de agosto al $1^{\circ}$ de septiembre de 2005 se prospectó isla Rupert, con el objeto de establecer la distribución de las colonias y realizar una estimación de su tamaño poblacional, la isla fue dividida en 40 cuadrantes de acuerdo a una grilla prefijada sobre una fotografía aérea del islote, con puntos conspicuos de referencia (Fig. 1). Para los 31 cuadrantes a los cuales pudimos acceder, se realizaron cuadrículas de $100 \mathrm{~m}^{2}$, con distancias de $160 \mathrm{~m}$ entre ellas, ubicados mediante navegadores GPS.

En cada cuadricula o parcela se consideró:

- Total de nidos de pingüinos, separándolos en activos evidentes (con plumas y fecas) e inactivos.

- Composición y cobertura de árboles y arbustos, vivos y muertos

- Pendiente del terreno 


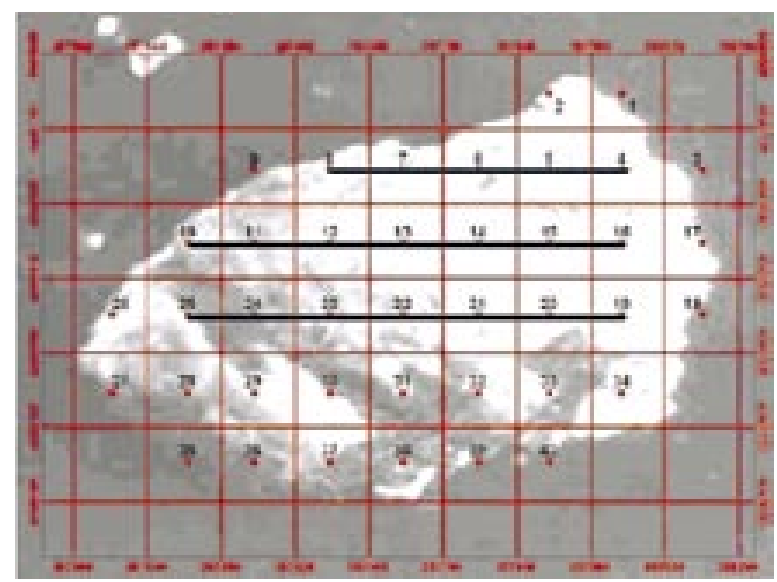

Fig. 1. Grilla prefijada con cuarenta cuadrantes y ubicación de puntos de muestreo con distancias de $160 \mathrm{~m}$ entre ellas, en los que se aplicaron en 2005 muestreos en cuadrículas de 10 x $10 \mathrm{~m}^{2}$ y las 3 transectas trazadas en 2007 por la meseta central de isla Rupert, horizontalmente en color negro.

Entre los días 13 al 19 de enero y desde el 7 al 13 de abril 2007, se procedió a realizar un remuestreo estratificado de la isla, con el objeto de definir de manera más precisa los límites de los parches con nidos y ajustar el estimador poblacional mediante la complementación de varias técnicas. Diseñamos un método nuevo, adecuado a las condiciones de isla Rupert para establecer los bordes o límites interiores de la colonia en torno al perímetro de la isla; dos observadores recorrieron el perímetro de la isla y deslindaron la colonia.

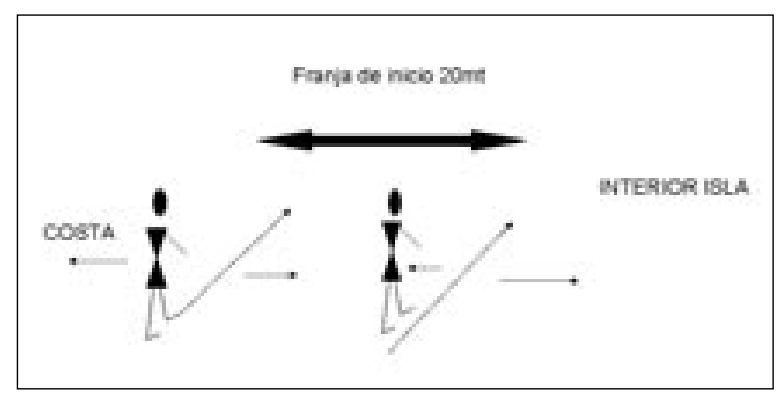

Fig. 2. Metodología empleada en deslinde de los bordes interiores o límites de la colonia de pingüinos de Magallanes en torno al perímetro de la isla Rupert.

Uno de ellos se dispuso en el interior y otro en el exterior o periféricamente, más cercano a la costa, separados por una distancia de 20 metros aproximadamente. Para ello caminaron en forma paralela a la costa, de modo tal que el observador periférico dejó siempre los nidos entre él y la costa y/o en la línea de avance. El segundo observador revisó la posible existencia de nidos en la parte más alta $e$ interior de la isla. Cuando el segundo observador encontró la presencia de un nido, el observador periférico se aproximó, anotando las coordenadas del nido de modo que entre ambos observadores se fue extiendo una franja sin nidos (Fig. 2). Las coordenadas de los nidos fueron obtenidas con un GPS modelo Garmin 12, considerándose el número de pingüinos adultos, polluelos, huevos y el tipo de la vegetación cercana al nido. Los datos de las coordenadas fueron ingresadas al software
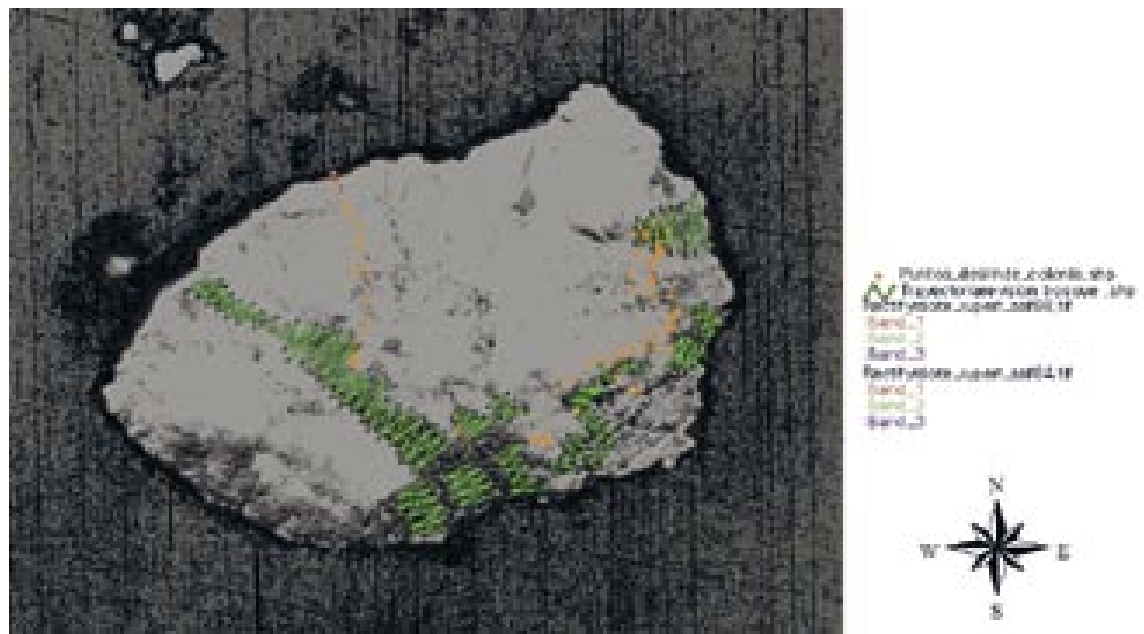

Fig. 3. Se indica con puntos la ubicación de nidos a lo largo de la ruta seguida de deslinde de los bordes interiores o límites de la colonia de pingüinos de Magallanes. en torno al perímetro de la isla Rupert. En trazo continuo se indica las rutas en zigzag seguidas para revisión de colonia al interior de bosques de poca visibilidad. 

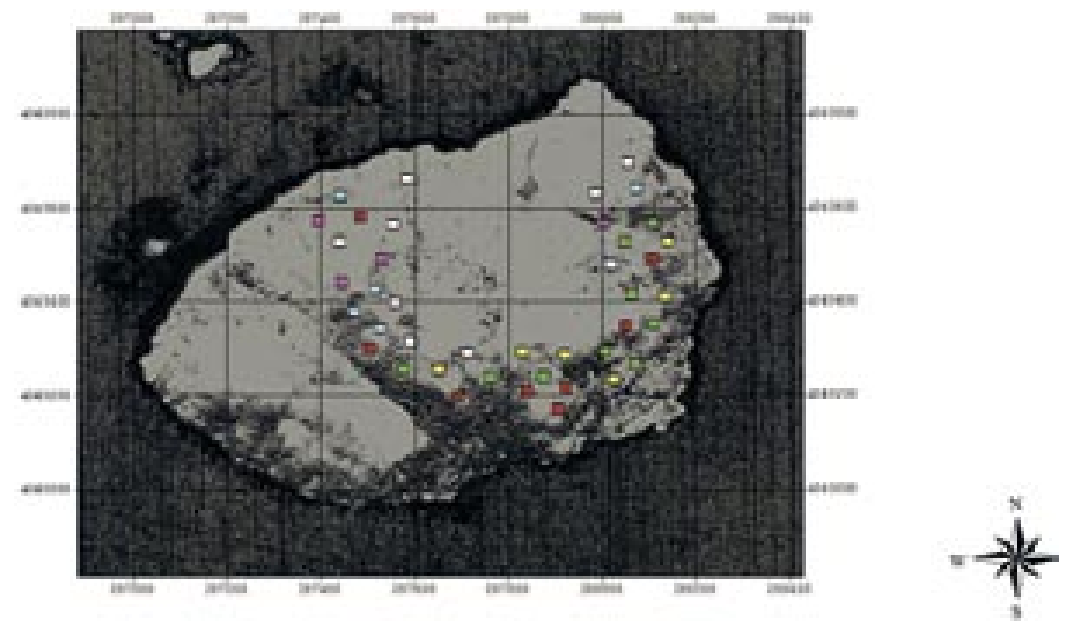

Fig. 4. Mapa con la distribución y ubicación de 40 cuadrantes de $100 \mathrm{~m}^{2}$ lanzados a $25 \mathrm{~m}$ de distancia, dentro de las colonias de isla Rupert. Las densidades de nidos activos se representa con diferentes colores, desde un máximo de 5 nidos $\square, 4$ nidos $\square, 3$ nidos $\square, 2$ nidos $\square$, 1 nido $\square$ y un mínimo de 0 nidos x 100 m² $^{2}$

computacional Arc view 3.2. Como complemento al método anterior se realizaron tres transectas para detectar nidos activos por el centro de la isla, zona muestreada también en 2005, sin evidencia de nidos. Las transectas se iniciaron en la costa de los cuadrantes 4, 16, 19 y finalizadas en la costa opuesta en los cuadrantes $(8,10,25)$ (Fig. 1).

Dentro de los bosques de isla Rupert la visibilidad es mínima a más de tres metros, por lo que para la estimación de la densidad de nidos en su interior, se revisaron las zonas de bosques vivos y muertos, siguiendo rutas en zigzag (Fig. 3) en los días 7 al 13 de abril 2007. Para comenzar a trazar esta ruta se eligió una ladera del bosque y se caminó hasta llegar a la ladera opuesta, y se retornó con una separación entre los dos puntos de la misma ladera de aproximadamente cinco metros, revisando y marcando en la trayectoria la existencia de nidos activos. En la prospección de abril de 2007 se escogieron al azar 40 cuadrantes de $100 \mathrm{~m}^{2}(10$ x $10 \mathrm{~m})$ cada 25 metros de distancia dentro de las zonas de colonias delimitadas con las técnicas mencionadas anteriormente (Fig. 4). El tamaño de los cuadrantes se definió siguiendo la metodología empleada para las colonias del pingüino de Magallanes de cabo Dos Bahías y cabo Vírgenes, Argentina (Capurro et al.
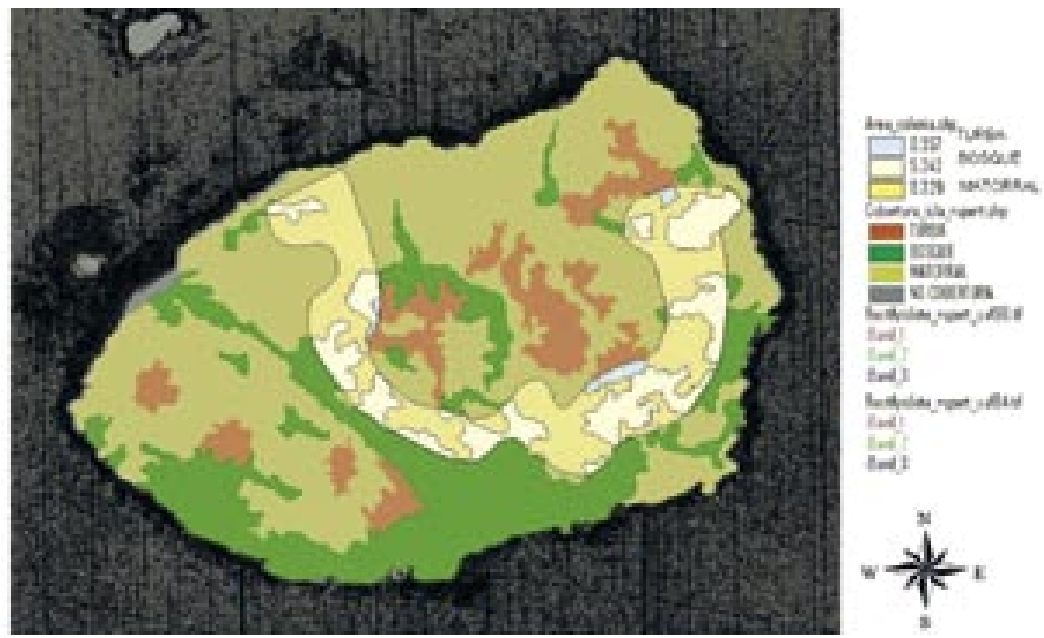

Fig. 5. Mapa con el área ocupada por nidos activos de la colonia del pingüino de Magallanes y áreas ocupadas por las comunidades vegetacionales de isla Rupert. 
TABLA 1: número de nidos, nidos activos e inactivos totales de pingüino de Magallanes por cuadrante ocupado.

\begin{tabular}{|c|c|c|c|}
\hline cuadrantes & $\mathrm{n}$ nidos & nidos activos & no activos \\
\hline 1 & 3 & 2 & 1 \\
\hline 2 & 4 & 3 & 1 \\
\hline 4 & 2 & 0 & 2 \\
\hline 5 & 1 & 1 & 0 \\
\hline 6 & 2 & 0 & 2 \\
\hline 7 & 4 & 1 & 3 \\
\hline 8 & 1 & 1 & 0 \\
\hline 9 & 7 & 2 & 5 \\
\hline 10 & 3 & 0 & 3 \\
\hline 11 & 3 & 2 & 1 \\
\hline 12 & 2 & 2 & 0 \\
\hline 13 & 5 & 3 & 2 \\
\hline 14 & 4 & 0 & 4 \\
\hline 15 & 7 & 4 & 3 \\
\hline 16 & 7 & 5 & 2 \\
\hline 17 & 8 & 3 & 5 \\
\hline 19 & 4 & 3 & 1 \\
\hline 20 & 9 & 4 & 5 \\
\hline 21 & 7 & 5 & 2 \\
\hline 22 & 3 & 3 & 0 \\
\hline 23 & 5 & 4 & 1 \\
\hline 24 & 5 & 3 & 2 \\
\hline 25 & 4 & 3 & 1 \\
\hline 26 & 5 & 5 & 0 \\
\hline 27 & 7 & 4 & 3 \\
\hline 28 & 7 & 5 & 2 \\
\hline 29 & 10 & 4 & 6 \\
\hline 30 & 8 & 3 & 5 \\
\hline 31 & 7 & 5 & 2 \\
\hline 32 & 5 & 4 & 1 \\
\hline 33 & 4 & 0 & 4 \\
\hline 34 & 9 & 3 & 6 \\
\hline 35 & 12 & 5 & 7 \\
\hline 36 & 9 & 4 & 5 \\
\hline 37 & 1 & 1 & 0 \\
\hline 38 & 3 & 2 & 1 \\
\hline 39 & 3 & 0 & 3 \\
\hline 40 & 5 & 0 & 5 \\
\hline total & 195 & 99 & 96 \\
\hline
\end{tabular}

1988, Gandini et al. 1997). Los cuadrantes fueron demarcados por medio de estacas de $1,5 \mathrm{~m}$, donde se incluyó el registro de las siguientes variables: nidos activos y no activos, tipo de vegetación donde se encontraba el nido, recolección de muestras de vegetación y fotografías. La pendiente fue obtenida mediante un clinómetro.

Durante las prospecciones realizadas a fines de agosto de 2005 se contabilizó un total de 101 nidos y 52 de ellos fueron reconocidos como nidos activos por la presencia de plumas en buen estado. El rango de la densidad de nidos activos registrado fue de 0 nidos $/ 100 \mathrm{~m}^{2}$ a un máximo de 23 nidos $/ 100 \mathrm{~m}^{2}$. En la tabla 1 se indica el número de nidos contabilizados para cada cuadrante. Sólo en un total de 11 cuadrantes (35,5\% del área muestreada) se encontraron evidencia de nidos activos de pingüinos. Los nidos se distribuyeron espacialmente en sectores con mayor cobertura arbórea y arbustiva, protegidos, correspondientes a la costa este y sur de la isla (Fig. 4). En tanto que en aquellos sectores en que la pendiente fue superior a $45^{\circ}$ aproximadamente, generalmente abiertos y de mayor altitud, que existen en la cara norte de la isla y también en su meseta central, no se encontraron nidos. La densidad promedio estimada de nidos, activos e inactivos, en los 11 cuadrantes fue de 8,6 nidos $/ 100 \mathrm{~m}^{2}$. Considerando que un $35,5 \%$ de la superficie de la isla habría estado ocupada por nidos de pingüinos, se obtiene un total de 20.455 nidos. No obstante, si se consideran sólo los nidos activos, arroja cerca de 4,7 nidos activos $/ 100 \mathrm{~m}^{2}$, lo cual asumiendo un $35,5 \%$ de la superficie ocupada, da un total para el islote de 11.250 nidos activos, lo que se traduciría en un total de 22.500 pingüinos adultos reproductivos en isla Rupert durante el 2005.

El área efectiva ocupada por la colonia de pingüinos de Magallanes en enero de 2007, fue de 14 hectáreas en toda la isla Rupert (Tabla 2, Fig. 5), equivalente al 19,4\% de la superficie total de la isla.

TABLA 2: Perímetro en metros, y del área en hectáreas de la colonia de pingüino de Magallanes en 2007, indicando las superficies de las comunidades vegetacionales que se encuentran al interior del área de la colonia calculadas por el software Arc, View 3.2.

\begin{tabular}{|c|c|c|c|}
\hline & Total isla Rupert & \multicolumn{2}{|c|}{ Colonia Pingüinos de Magallanes } \\
\hline COMUNIDADES & Área hectáreas & Perímetros metros & Area hectáreas \\
\hline BOSQUE & 22.452 & 4.815 .066 & 5.242 \\
\hline MATORRAL & 42.332 & 5.751 .794 & 8.329 \\
\hline TURBA & 7.581 & 641.833 & 0.357 \\
\hline Total & 72.365 & & 13,928 \\
\hline
\end{tabular}


TABLA 3: Densidades de los nidos activos e inactivos de pingüino de Magallanes, considerando los cuadrantes ocupados y no ocupados por nidos.

\begin{tabular}{|c|c|c|c|}
\hline $\begin{array}{l}\text { descripción estadística } \\
\text { n de nidos año } 2007\end{array}$ & & & \\
\hline variable $\mathrm{n}$ de nidos & $\mathrm{N}$ cuadrantes & promedio & Desviación estándar \\
\hline $\mathrm{N}$ de nidos & 40 & 4,875 & 0,455 \\
\hline variable & mínimo & máximo & \\
\hline $\mathrm{n}$ de nidos & 0 & 12 & \\
\hline descripción estadística & $\mathrm{N}$ & promedio & Desviación estándar \\
\hline nidos activos & 40 & 2,475 & 0,28 \\
\hline variable & mínimo & máximo & \\
\hline $\mathrm{n}$ de nidos & 0 & 5 & \\
\hline descripción estadística & $\mathrm{N}$ & promedio & Desviación estándar \\
\hline nidos inactivos & 40 & 2,4 & 0,318 \\
\hline variable & mínimo & máximo & \\
\hline nidos inactivos & 0 & 7 & \\
\hline
\end{tabular}

Al igual que en 2005, los pingüinos no utilizaron, o lo hicieron en muy baja densidad, las áreas expuestas al noroeste, la meseta central y los sectores costeros de alta pendiente. Los sitios de nidificación se encuentran preferentemente asociados a las comunidades de matorrales, representando el $19 \%$ de la superficie total de esta comunidad en esta isla (Fig. 5, Tabla 2). En enero de 2007 se obtuvieron densidades promedio de 4, 88 nidos/ 100 m-2 o 488 nidos /ha con desviación estándar (DE) de 0,44 (Tabla 3). Si se consideran sólo los nidos activos, la densidad promedio fue de 2,48 nidos/100 m-2 o 248 nidos /ha con una DE de 0,28. Lo cual implica una estimación gruesa de 3.472 nidos activos en las 14 hectáreas ocupadas, y que se traduciría en un total aproximado de 6.944 pingüinos adultos reproductivos en isla Rupert en el verano de ese año.

El conocimiento de la ecología del pingüino de Magallanes se basa en estudios desarrollados principalmente en el extremo oriental de su gradiente de distribución (García et al. 2002), ambientes con pluviosidades anuales inferiores a los $430 \mathrm{~mm}$ no existiendo estudios publicados sobre patrones de abundancia y selección de ambientes en colonias reproductivas del sector occidental de su distribución, que incluye los fiordos patagónicos chilenos, en que las precipitaciones superan los $2.000 \mathrm{~mm}$ (Santana \& Zamora 1979, Schneider et al. 2003).

Nuestros resultados sobre la distribución y abundancia de pingüinos en isla Rupert entregan evi- dencia cuantitativa y cualitativa sobre la ocurrencia de un patrón de distribución agregada dentro de sectores protegidos de la isla, el que se ha conservado entre 2005 y 2007, excluyendo áreas expuestas abiertas y la meseta central de la isla, siendo esta última ocupada por salteadores (Stercorarius chilensis). La colonia ocupa preferentemente las comunidades de matorrales de matas altas y ecotonos de bosque, lo que podría vincularse a protección frente a la exposición del viento, lluvias y predadores como los salteadores. Algo similar encontraron Gandini et al. (1997) en la colonia de cabo Vírgenes, en la costa Atlántica, en donde las áreas con mayor cobertura arbustiva y de mayor altura favorecía el éxito reproductivo de esta especie y en la colonia de punta Tombo, en donde el mayor éxito se obtuvo en los lugares con mayor cobertura de la vegetación. Es interesante destacar que en isla Rupert no se registraron nidos activos en las áreas con bosques densos, en la costa protegida de la isla. No obstante sí se registraron nidos no activos, lo que indicaría un abandono del área, el cual no estaría vinculado a la perdida de cobertura de vegetación. No ha sido posible establecer las causas de lo anterior, pero sugerimos un probable efecto de inundación de nidos en base al relieve y baja pendiente de esas áreas.

En cuanto a la estimación del tamaño poblacional para el año 2007 de 6.944 individuos comparado con 22.500 ejemplares de la temporada del 2005, puede explicarse por diferencias meto- 


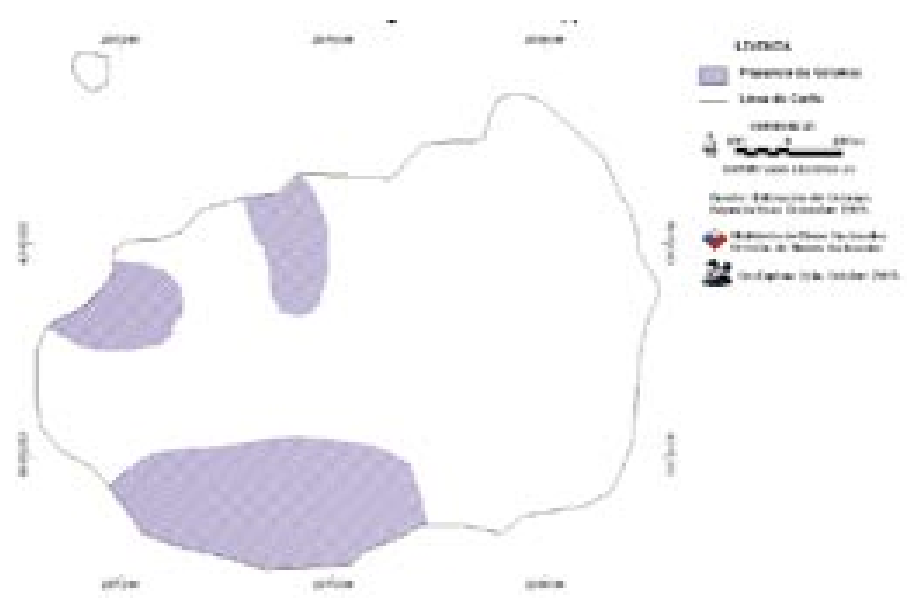

Fig. 6. Mapa con el área ocupada por la colonia del pingüino de Magallanes de isla Rupert en 1989 de acuerdo a Soto (1990)².

dológicas, pues en 2007 se realizó un muestreo más exhaustivo de la isla y en especial una mayor cantidad de cuadrantes dentro de los límites de la colonia, lo que permitió la obtención de datos más fidedignos, aumentando el grado de certeza. Además, ambos muestreos fueron realizados en estaciones distintas; los valores del 2005 se tomaron a fines de agosto, cuando las aves regresan en grandes cantidades a las colonias para iniciar la temporada reproductiva. Los registros del 2007 correspon- den a enero y abril, cuando la reproducción está terminando. Durante el inicio de la estación reproductiva, se espera una mayor actividad y presencia de aves en la colonia, ya que ambos miembros de la pareja están en las proximidades de los nidos y están presentes aquellas parejas cuya reproducción no será exitosa.

Según el estudio realizado por Soto (1990), durante la prospección realizada por él, en enero de 1989, los pingüinos se distribuían en tres colonias

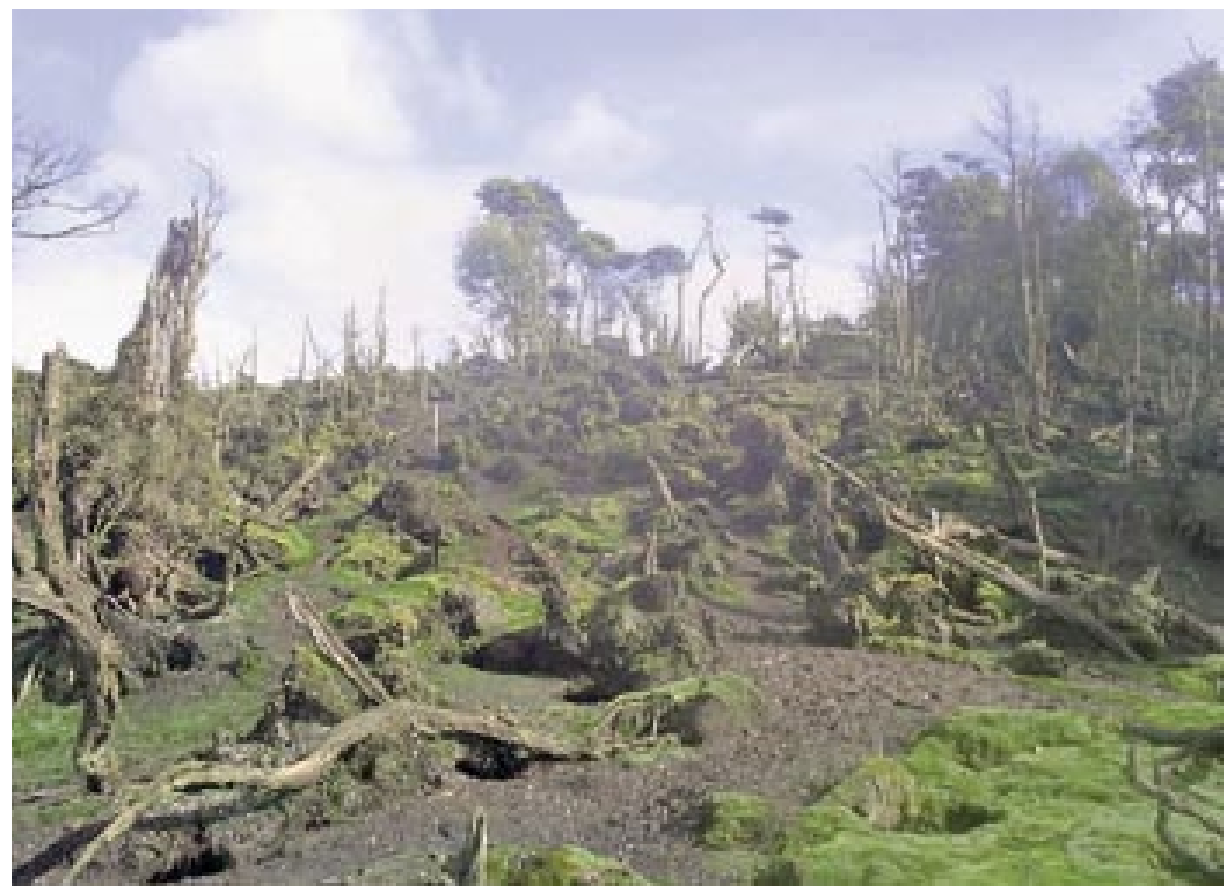

Fig. 7. Efecto erosivo producido por la colonia del pingüino de Magallanes en un bosque de coigüe en isla Rupert. 
(Fig. 6) ubicadas en el lado sur - suroeste de la isla, asociadas a bosques representadas por especies dominantes de coigüe (Nothofagus betuloides), canelo (Drymis winterii), ciprés (Pilgerodendrum uvifera), leñadura (Maytenus magellanica), y otras especies como Michay, romerillo, frutilla del diablo, junquillo, Poa flabellata, helechos como Blechnum magellanicum y en laderas de pendiente pronunciada y de alta densidad arbórea y arbustiva. Este autor estima la existencia de 3.764 nidos de pingüinos, con densidades que fluctúan de 4,5 a 22,7 nidos $/ 100 \mathrm{~m}^{2}$ y un mínimo de 2.246 pingüinos adultos. Las densidades estimada para las tres áreas o parches de nidificación fueron de 3,2; 6,2 y 7,1 nidos activos $/ 100 \mathrm{~m}^{2}$, lo cual está dentro del rango estimado por este estudio. No obstante, el tamaño de la población estimada para toda la isla fue de 2.248 adultos, un tamaño significativamente inferior al aportado por el presente estudio. Esta diferencia radicaría en un cambio en la distribución y también en el área total ocupada por los pingüinos de Magallanes. En 1989 habrían ocupado un total de 1,9 hectáreas y en 2007 unas 14 hectáreas. Sin embargo, Zambrano (citado por Soto 1990) ${ }^{2}$ señala que en la temporada 87/88 la colonia era más extensa que en 1989. Soto (1990)² confirma la observación de grandes extensiones de bosque seco de coigüe y canelo, con arbustos del género Senecio spp. y vegetación herbácea de Poa flabellata, comunidades vegetales típicas de ambientes abandonados por pingüinos (Dollenz 1990). Por ello no es posible en este momento indicar si el aumento en relación a 1989 corresponde a una tendencia en progresión o a fluctuaciones de otra naturaleza.

Nuestros resultados distinguirían de manera cualitativa, un posible cambio en el patrón de distribución espacial de las colonias con respecto a lo observado en 1989. En el futuro se hace necesario estudios más detallados sobre la relación que pueda tener la vegetación y el efecto erosivo de modificación del sustrato aportado por las deyecciones de los pingüinos descrito por Pisano (1971) y por Pisano \& Schlatter (1981) sobre la vegetación y en particular sobre un menor efecto de protección o refugio de ésta para la nidificación (Fig. 7). De acuerdo a Pisano (1971) los pingüinos de Magallanes participarían activamente en los estados sucesionales de la vegetación de los bosques sub-antárticos.

\section{AGRADECIMIENTOS}

Los autores agradecen a WhaleSound Ltda., Fundación Yubarta, Universidad de Magallanes. Este estudio fue parcialmente financiado por el Ministerio de Bienes Nacionales. El trabajo de J. Gibbons en Chile fue financiado parcialmente por fondos de la "Acción Integrada Universidad Complutense de Madrid - Universidad de Magallanes". Agradecemos la ayuda en terreno a Gloria Gallegos, de análisis estadístico a Derek Corcoran, de interpretación vegetacional a Juan Marcos Henríquez y de diseño de figuras a Esteban Sagredo. Los autores agradecen los importantes aportes que realizaron los dos revisores anónimos, quienes demostraron un gran conocimiento de la ecología reproductiva de esta especie.

\section{LITERATURA CITADA}

Bingham. M. 2002 Decline of Falklands penguins in the presence of a commercial fishing industry. Revista Chilena de Historia Natural 75: $805-818$.

Bingham. M. \& T. Martin 2008. Magellanic Penguin (Spheniscidae) monitoring results for Magdalena island (Chile) 2000 - 2008. Anales Instituto Patagonia (Chile) 36 (2): 19-32.

Boersma P.D. 1997. Magellanic penguins decline in south Atlantic. Penguin conservation 10:1-25.

Boswall J. \& D Maclver 1975. The Magellanic Penguin Spheniscus magellanicus. (271 -305) In Bernard Stonehouse (Ed.) The biology of Penguins. Macmillan Londres. 555 pp.

Brian G. Walker, P.D. Boersma \& J.C. Wingfield 2004. Physiological condition in Magellanic penguins: Does it matter if you have to walk a long way to your nest? The Condor 106: 696-701

Capurro, A., E. Frere, M. Gandini, P. Gandini, T. Holik, V. Litchstchein \& P.D. Boersma 1988. Nest density and population size of Magellanic Penguin (Spheniscus magellanicus) at Cabo Dos Bahias, Argentina. Auk 105: 585- 588.

Cruz, I. 2001. Los pingüinos como presas durante el Holoceno. Información biológica, fósil y arqueológica para discutir su disponibilidad 
en el sur de la patagonia. Arqueofauna 10: 99-112.

Frere. E., P. Gandini \& P.D. Boersma 1992. Effects of nest type and location on reproductive success of the Magellanic Penguin (Spheniscus magellanicus). Marine Ornithology 20:1-6.

Gandini P., E. Frere \& P.D. Boersma 1997. Efectos de la calidad de hábitat sobre el éxito reproductivo del pingüino de Magallanes (Spheniscus magellanicus) en Cabo Vírgenes, Santa Cruz, Argentina. Ornitología Neotropical 8:37- 48.

Gandini P., E. Frere \& P.D. Boersma 1999. Nest concealment and its relations to predation and reproductive success in the Magellanic Penguin at its southernmost Continental colony. Ornitología Neotropical 10:145-150.

García, P., P. Yorio, P.D. Boersma, H. del Valle \& M. Bertellotti 2002. Habitat use and breeding distribution of Magellanic penguins in northern San Jorge Gulf, Patagonia, Argentina. Auk 119: 233-239.

Kusch, A., M. Marín, D. Oheler \& S. Drieschman 2007. Notas sobre la avifauna de isla Noir $\left(54^{\circ} 28^{\prime} \mathrm{S}-73^{\circ} 00^{\prime} \mathrm{W}\right)$. Anales Instituto Patagonia (Chile) 35(2): 61-66.

Pisano, E. 1971. Estudio ecológico preliminar del Parque Nacional "Los pingüinos" (Estrecho de Magallanes). Anales Instituto Patagonia (Chile) 1: 77-92.

Pisano, E. 1972. Observaciones Fito-ecológicas en las Islas Diego Ramírez. Anales Instituto Patagonia (Chile), 3(1-2):162-169.

Pisano, E \& R.P. Schlatter 1981.Vegetación y flora de las islas Diego Ramírez (Chile) II. Comunidades vegetales vasculares. Anales Instituto Patagonia (Chile) 12:196-204.

Santana A. \& E. Zamora 1979. Características climáticas de la costa occidental de la Patagonia entre las latitudes $46^{\circ} 40^{\prime}$ y $56^{\circ} 30^{\prime}$ S. Anales Instituto Patagonia (Chile) 10:110-144.
Schneider C., M. Glaser, R. Kilian, A. Santana, N. Butorovic \& G. Cassasa 2003. Weather observations across the southern Andes at 53․ S. Phisical Geography 24 (2): 97 -119 .

Scolaro. J. \& A. Reyna 1984. Principales efectos ecológicos que afectan la nidificación del pingüino de Magallanes (Spheniscus magellanicus) en la colonia de Punta Tombo. Contribución Centro Nacional Patagónico (Argentina). 97:1-14.

Simeone A. \& R. Schlatter 1998. Threats to a mixed-species colony of Spheniscus penguins in Southern Chile. Colonial Waterbirds 21:418-421.

Stokes. D. L \& P.D. Boersma 1991. Effects of substrate on the distribution of Magellanic penguin (Spheniscus magellanicus). Auk 108:923-933.

Stokes, D.L \& P.D. Boersma 2001. Nesting density and reproductive success in a colonial seabird, the Magellanis penguin. Ecología. 81:2878-2891.

Schiavini, A., P .Yorio, P. Gandini, A. Raya \& P.D. Boersma 2005. Los pingüinos de las costas Argentinas: Estado poblacional y conservación. Hornero 20(1): 5-23

Schlatter R.P. \& G.M. Riveros 1997. Historia natural del archipiélago Diego Ramírez, Chile. Serie Científica INACH 47: 87-112.

Venegas, C. 1999. Estado de conservación de las especies de pingüinos en la región de Magallanes, Chile. Estudios Oceanológicos 18:45-56.

Venegas, C. 1978. Pingüinos de Barbijo (Pygoscelis antarctica) y Macaroni (Eudyptes chrysolophus) en Magallanes. Anales Instituto Patagonia (Chile) 9:180-183.

Venegas, C. 1994. Aves de Magallanes. Ediciones de la Universidad de Magallanes. Punta Arenas (Chile). 158 pp. 
